\title{
Testing CGR against High Redshift Observations
}

\author{
John G. Hartnett \\ School of Physics, the University of Western Australia, \\ 35 Stirling Hwy, Crawley 6009 WA Australia \\ john@physics.uwa.edu.au \\ Firmin J. Oliveira \\ Joint Astronomy Centre Hilo, Hawai‘i 96720 \\ firmin@jach.hawaii.edu
}

\begin{abstract}
Several key relations are derived for Cosmological General Relativity which are used in standard observational cosmology. These include the luminosity distance, angular size, surface brightness and matter density. These relations are used to fit type Ia supernova ( $\mathrm{SNe}$ Ia) data, giving consistent, well behaved fits over a broad range of redshift $0.1<z<2$. The best fit to the data for the local density parameter is $\Omega_{m}=0.0401 \pm$ 0.0199. Because $\Omega_{m}$ is within the baryonic budget there is no need for any dark matter to account for the SNe Ia redshift luminosity data. From this local density it is determined that the redshift where the universe expansion transitions from deceleration to acceleration is $z_{t}=1.095_{-0.155}^{+0.264}$. Because the fitted data covers the range of the predicted transition redshift $z_{t}$, there is no need for any dark energy to account for the expansion rate transition. We conclude that the expansion is now accelerating and that the transition from a closed to an open universe occurred about $8.54 \mathrm{Gyr}$ ago.
\end{abstract}

\section{Introduction}

Carmeli's cosmology, also referred to as Cosmological General Relativity (CGR), is a space-velocity theory of the expanding universe. It is a description of the universe at a particular fixed epoch of cosmic time $t$. In CGR time is measured from the present back toward the beginning. The theory assumes the Hubble law as fundamental. The observables are the coordinates of Hubble; proper distance and velocity of the expansion of the Universe. In practice, not velocity but redshift is used. CGR incorporates this basic law into a general $4 D$ Riemannian geometrical theory satisfying the Einstein field equations (Ref. [2], appendix A).

In order to compare the theoretical predicted redshift distance modulus relation of CGR with the distance modulii derived from type Ia supernova data, 
firstly, luminosity distance must be determined in this theory. Secondly, we need to model correctly the variation of matter density with redshift in the Universe. In the following, we determine a few key relations that are used in the subsequent analysis. Then we compare the theoretical distance modulii with those measured, resulting in a good fit without the need to assume the existence of dark energy or dark matter.

\section{Luminosity distance}

Suppose $L$ is the total energy emitted per unit time by a source galaxy at the epoch $t$ (that is, in the rest frame of the galaxy) to be received by an observer at the present time $t=0$. Therefore we can write

$$
d L=L I(\lambda) d \lambda
$$

where $I$ is its (normalized) intensity distribution - a function of wavelength $\lambda$. In CGR, times at cosmological distances add according to a relativistic addition law [3] when referred to the observer at $t=0$. Hence instead of the time interval $\Delta t$, we get

$$
\Delta t \rightarrow \frac{t+\Delta t}{1+\frac{t \Delta t}{\tau^{2}}}-t=\Delta t\left\{1-\frac{t^{2}}{\tau^{2}}\right\},
$$

where $\tau \approx H_{0}^{-1}$ is the Hubble-Carmeli time constant. From this it can be shown [15] that the luminosity $L_{0}$ of a source at the present time is related to the luminosity $L$ of an identical source which emitted at time $t$ by

$$
L_{0}=L\left\{1-\frac{t^{2}}{\tau^{2}}\right\}
$$

For the source at distance $r$, redshift $z$, emission wavelength $\lambda_{0} /(1+z)$ and the luminosity (3), it is straight forward to show [17] that the observed flux integrated over all wavelengths is

$$
\mathcal{F}_{b o l}=\frac{L_{b o l}}{(1+z)^{2}}\left\{1-\frac{t^{2}}{\tau^{2}}\right\} \frac{1}{4 \pi r^{2}}=\frac{L_{b o l}}{4 \pi \mathcal{D}_{L}^{2}},
$$

where $L_{b o l}$ is the absolute bolometric luminosity of the source galaxy. Therefore the luminosity distance $\mathcal{D}_{L}$ in CGR is expressed as

$$
\mathcal{D}_{L}=r(1+z)\left\{1-\frac{t^{2}}{\tau^{2}}\right\}^{-1 / 2}
$$

It is clear that this expression for the luminosity distance in CGR when compared to that in the FRW theory has the extra factor $\left(1-t^{2} / \tau^{2}\right)^{-1 / 2}$. Hence we expect the luminosity distance to be greater in CGR than in FRW theory. 
Figure 1: Angular size shown as a function of redshift for both the FRW model (solid line) with a deceleration parameter $q_{0}=1 / 2$ or $\Omega_{m}=1$ and the CGR model with $\Omega_{m}=0.04$ (broken line)

\section{Angular size}

The line element in CGR [9]

$$
d s^{2}=\tau^{2} d v^{2}-\left(1+(1-\Omega) \frac{r^{2}}{c^{2} \tau^{2}}\right)^{-1} d r^{2}-r^{2}\left(d \theta^{2}+\sin ^{2} \theta d \phi^{2}\right),
$$

represents a spherically symmetric isotropic universe. See Ref $[2,9]$ for details. The expansion is observed at a definite time and therefore $d t=0$ and hence doesn't appear in (6). Carmeli solved (6) with the null condition $d s=0$ and isotropy $(d \theta=d \phi=0)$ from which it follows that the proper distance $r$ in spherically symmetric coordinates can be written as

$$
\frac{r}{c \tau}=\frac{\sinh (\beta \sqrt{1-\Omega})}{\sqrt{1-\Omega}},
$$

where $\beta=t / \tau=v / c$ and $\Omega$ is matter density, a function of redshift $z$. Also $\beta$ can be written as a function of redshift

$$
\beta=\frac{(1+z)^{2}-1}{(1+z)^{2}+1} .
$$

Now in CGR there is no scale factor like in the FRW theory but we can similarly define an expansion factor as $(1+z)^{-1}$. If we then make the substitution for the matter density $\Omega=\Omega_{m}(1+z)^{3}$, where $\Omega_{m}$ is the matter density at the current epoch, the proper distance (7) can be rewritten as a function of $(1+z)$,

$$
r=c \tau \sinh \left(\beta \sqrt{1-\Omega_{m}(1+z)^{3}}\right) / \sqrt{1-\Omega_{m}(1+z)^{3}} .
$$

For a proper comparison with FRW theory we must use the FRW equivalent of $r /(1+z)$, which is the Hubble distance $D_{1}$ when the light we observe left the galaxy at redshift $z$ and is given by

$$
D_{1}=\frac{2 c H_{0}^{-1}}{(1+z)}\left\{1-\frac{1}{\sqrt{1+z}}\right\}
$$

where a deceleration parameter $q_{0}=1 / 2$ has been used. The angular size of the source galaxy in FRW theory is

$$
\Delta \theta=\frac{d}{D_{1}}
$$

where $d$ is the actual diameter of the source galaxy and the angular distance $D_{1}$ is taken from (10). 
In CGR the angular distance $\mathcal{D}_{A}$ is defined identically with (11)

$$
\Delta \theta=\frac{d}{\mathcal{D}_{A}},
$$

where the functional form for $\mathcal{D}_{A}$ is determined by its relationship to the luminosity distance $\mathcal{D}_{L}$. To show how $\mathcal{D}_{L}$ and $\mathcal{D}_{A}$ are related we look at the flux $F_{\theta}$ from a distant source of extent $d$ which subtends an angle $\Delta \theta$ on the sky [25]

$$
F_{\theta}=\Delta \theta^{2} \sigma T_{o}^{4},
$$

where $\sigma$ is the Stephan-Boltzmann constant and $T_{o}$ is the observed temperature of the source. Equating fluxes from (4) and (13), substituting for $\Delta \theta$ from (12) and substituting $L_{b o l}=4 \pi d^{2} \sigma T_{e}^{4}$ with $T_{e}$ the source temperature we get

$$
\frac{T_{e}^{4}}{\mathcal{D}_{L}^{2}}=\frac{T_{o}^{4}}{\mathcal{D}_{A}^{2}} .
$$

Since for a blackbody at temperature $T$ the radiation with average wavelength $\lambda$ has energy $h c / \lambda=k T$ where $k$ is Boltzmann's constant and since the wavelength varies with redshift as $(1+z)$ this implies $T_{o}=T_{e} /(1+z)$. We assume that this holds even for a galaxy source which may not be a perfect blackbody. Then (14) simplifies to

$$
\mathcal{D}_{L}=\mathcal{D}_{A}(1+z)^{2} .
$$

This relation is the same as that for FRW. Hence the angular size of a source galaxy in CGR can be found

$$
\Delta \theta=\frac{d}{\mathcal{D}_{A}}=\frac{d(1+z)}{r}\left\{1-\frac{t^{2}}{\tau^{2}}\right\}^{1 / 2}
$$

where (5) and (15) have been used.

Substituting (9) in (16) produces gravitational effects on the angular size that can be called lensing. We have plotted in Fig. 1 the dependence of angular size $\Delta \theta$ on redshift $z$ for CGR using (9) in (16) but instead with the density function $\Omega(z)$ determined by Oliveira and Hartnett [18]. That density expression replaces the simple form in (9) and better characterizes the universe at high redshifts.

In order to compare theories independently of the constants $d, c$ and $\tau \approx$ $H_{0}^{-1}$, we plot $\Delta \theta(z) / \Delta \theta(0.01)$ for both FRW and CGR theories. It is quite clear from Fig. 1 that for redshifts $z \leq 0.2$ the two models are in reasonable agreement but in general $\Delta \theta_{F R W} \neq \Delta \theta_{C G R}$. For $z>0.2$ the details depend heavily on the parameters of the models chosen.

\section{Surface brightness}

To determine the effect of redshift variation on apparent surface brightness $B$ of a source we need to calculate the observed flux $\mathcal{F}_{\text {bol }}$ per unit solid angle $\Theta$,

$$
B=\frac{\mathcal{F}_{b o l}}{\Theta}
$$


where for a source diameter of $d$ and source angular distance $\mathcal{D}_{A}$, the solid angle $\Theta$ is given by

$$
\Theta=\frac{\pi(d / 2)^{2}}{\mathcal{D}_{A}^{2}} .
$$

It follows from (4), (15), (17) and (18) that the apparent surface brightness

$$
B=\frac{\mathcal{F}_{b o l}}{(\pi / 4) d^{2} / \mathcal{D}_{A}^{2}}=\frac{L_{b o l}}{\pi^{2} d^{2}}(1+z)^{-4},
$$

which is the same as the usual FRW expression, the same $(1+z)^{-4}$ dependence Tolman [23] produces using standard cosmology.

\section{Density}

In terms of the phase space expansion history, the universe at time $t$ has a total relativistic mass $M$ and a total volume $V$. The expansion is assumed to be symmetric so that the volume $V$ is spherical. The average matter density $\rho$ is

$$
\rho=\frac{M}{V} .
$$

The total relativistic mass of matter $M$ in Cosmological Special Relativity [2] at cosmic time $t$ is

$$
M=\frac{M_{0}}{\sqrt{1-t^{2} / \tau^{2}}},
$$

where $M_{0}$ is the mass of the universe at the present epoch $t=0$.

The volume is taken to be that of a sphere

$$
V=\frac{4 \pi}{3} R^{3},
$$

where $R$ is the radius of the portion of the universe that just contains the mass $M$. In CGR, the distance $r$ is measured from the observer at the present epoch to the source rather than the other way, e.g. as is done in the Friedmann theory of cosmology. We assume that higher density corresponds to higher velocity and that the volume decreases as velocity increases. The radius $R$ of the universe is therefore taken to be

$$
R=c \tau-r,
$$

where the redshift distance relationship $r$ is given by (7).

$R$ is defined this way so that for $v=0, R(r=0)=c \tau$ is the radius of the sphere of the universe that just contains the mass of matter $M_{0}$. We define the average matter density parameter

$$
\Omega=\frac{\rho}{\rho_{c}},
$$


where $\rho_{c}=3 /\left(8 \pi \mathrm{G} \tau^{2}\right)$ is the critical density. An overall constraint is that, for $\Omega \geq 0$,

$$
1+\frac{(1-\Omega)}{c^{2} \tau^{2}} r^{2}>0 .
$$

From (20)-(24) the function for $\Omega$ is

$$
\Omega=\frac{\Omega_{m} / \sqrt{1-\beta^{2}}}{[1-\sinh (\beta \sqrt{1-\Omega}) / \sqrt{1-\Omega}]^{3}},
$$

where

$$
\begin{aligned}
\Omega_{m} & =\frac{\rho_{m}}{\rho_{c}}, \\
\rho_{m} & =\frac{M_{0}}{(4 \pi / 3)(c \tau)^{3}},
\end{aligned}
$$

where $\rho_{m}$ is the average matter density at the current epoch.

In the first order approximation where $\beta \ll 1, z \approx \beta$. Since $\sinh (x) \approx x$ for small $x,(26)$ can be written

$$
\Omega \approx \frac{\Omega_{m}\left(1+(1 / 2) \beta^{2}\right)}{(1-\beta)^{3}} \approx \Omega_{m}(1+z)^{3} .
$$

In the Friedmann-Robertson-Walker cosmologies, the matter density parameter $\Omega=\Omega_{m}(1+z)^{3}$ for all $\mathrm{z}$ in a dust dominated spatially flat universe, but this is not the case in the present theory where the density varies more strongly than $(1+z)^{3}$. This will produce significant results in the data analysis.

The derived relation (26) for $\Omega$ is transcendental. For fits to data it is more convenient to have a regular function, hence we use a second order approximation for $\Omega$, which is briefly described in appendix (A).

\section{$6 \quad$ Expansion transition redshift $z_{t}$}

In CGR the expansion has three basic phases: decelerating, coasting and finally accelerating, corresponding to density $\Omega>1, \Omega=1$, and $\Omega<1$, respectively [5]. What is the expected velocity and redshift of the transition from deceleration to acceleration? This phase shift occurs during the zero acceleration or coasting phase when $\Omega=1$. Taking (26) to the limit $\Omega \rightarrow 1$, $\operatorname{since} \sinh (x) \approx x$ for small $x$, yields

$$
\lim _{\Omega \rightarrow 1} \Omega=1=\frac{\Omega_{m} / \sqrt{1-\beta_{t}^{2}}}{\left(1-\beta_{t}\right)^{3}},
$$

which simplifies to

$$
\left(1-\beta_{t}\right)^{3} \sqrt{1-\beta_{t}^{2}}=\Omega_{m} .
$$

Solving (31) for $\beta_{t}$, the predicted redshift $z_{t}$ of the expansion transition is obtained from (8). 
Figure 2: The combined data sets of Riess et al and Astier et al. The solid line represents the statistically best fit curve with $a=0.2284$ and $\Omega_{m}=0.0401$ and the broken line represents the curve with $a=0.2284$ and $\Omega_{m}=0.263$

\section{Comparison with high-z type Ia supernova data}

The redshift distance relationship in CGR is given by (7) and $\Omega$ is evaluated from (46).

In order to compare the redshift distance relation with the high redshift SNe Ia data from Riess et al [20] and Astier et al [1], the proper distance is converted to magnitude as follows.

$$
m(z)=\mathcal{M}+5 \log \left[\mathcal{D}_{L}(z ; \Omega)\right],
$$

where $\mathcal{D}_{L}$ is the dimensionless "Hubble constant free" luminosity distance (5). Refer [19, 21]. Here

$$
\mathcal{M}=5 \log \left(\frac{c \tau}{M p c}\right)+25+M_{B}+a .
$$

The units of $c \tau$ are $M p c$. The constant 25 results from the luminosity distance expressed in $M p c$. However, $\mathcal{M}$ in (32) represents a scale offset for the distance modulus $\left(\mathrm{m}-M_{B}\right)$. It is sufficient to treat it as a single constant chosen from the fit. In practice we use $a$, a small free parameter, to optimize the fits. From (5), with $\beta=t / \tau$ the luminosity distance is given by

$$
\mathcal{D}_{L}\left(z ; \Omega_{m}\right)=\frac{r}{c \tau}(1+z)\left(1-\beta^{2}\right)^{-1 / 2}
$$

using (7), hence $r$ in units of $c \tau$. $\mathcal{D}_{L}$ is only a function of $\Omega_{m}$ and $z$.

The parameter $\mathcal{M}$ incorporates the various parameters that are independent of the redshift, $z$. The parameter $M_{B}$ is the absolute magnitude of the supernova at the peak of its light-curve and the parameter $a$ allows for any uncompensated extinction or offset in the mean of absolute magnitudes or an arbitrary zero point. The absolute magnitude then acts as a "standard candle" from which the luminosity and hence distance can be estimated.

The value of $M_{B}$ need not be known, neither any other component in $\mathcal{M}$, as $\mathcal{M}$ has the effect of merely shifting the fit curve (34) along the magnitude axis.

However by choosing the value of the Hubble-Carmeli constant $\tau=4.28 \times$ $10^{17} \mathrm{~s}=13.58 \mathrm{Gyr}$, which is the reciprocal of the chosen value of the Hubble constant in the gravity free limit $h=72.17 \pm 0.84$ (statistical) $k m \cdot s^{-1} \mathrm{Mpc}^{-1}$ (see Section 9.1) $\mathcal{M}=43.09+M_{B}+a$.

We use two SNe Ia data sets for the curved fitting analysis. The data are drawn from Table 5 of Riess et al [20], the Supernova Cosmology Project, and Tables 8 and 9 of Astier et al [1], the Supernova Legacy Survey (SNLS). Also 
Figure 3: Residuals vs redshift (on linear scale): the differences between the best fit curve with $\Omega_{m}=0.0401$ and $a=0.2284$ and the data of fig. 2. The mean of the residuals is $8.04 \times 10^{-5}$ when all errors are assumed equal and -0.0769 when weighted by published errors. The broken line represents the curve where $\Omega_{m}=0.263$

we combined the data sets of Riess et al and Astier et al and found the best statistical fit to all those data.

This is shown in fig. 2 along with the curve where $\Omega_{m}=0.263$, which is the value that Astier et al quote for the average matter density at the current epoch. Lastly, we take the residuals between the combined the data set of Riess et al and Astier et al and the best fit curve of fig. 2. This is shown in fig. 3, along with the curve that represents $\Omega_{m}=0.263$.

\section{Quality of curve fits}

In order quantify the goodness of the least squares fitting we have used the $\chi^{2}$ parameter which measures the goodness of the fit between the data and the theoretical curve assuming the two fit parameters $a$ and $\Omega_{m}$. Hence $\chi^{2}$ is calculated from

$$
\chi^{2}=\sum_{i=1}^{N} \frac{1}{\sigma_{i}^{2}}\left[(m-M)(z)_{i}-(m-M)\left(z_{o b s}\right)_{i}\right]^{2},
$$

where $N$ are the number of data; $(m-M)(z)$ are determined from (32) with fit values of $a$ and $\Omega_{m} ;(m-M)\left(z_{o b s}\right)$ are the observed distance modulus data at measured redshifts $z_{o b s} ; \sigma_{i}$ are the published magnitude errors. The values of $\chi^{2} / N\left(\approx \chi_{\text {d.o.f }}^{2}\right)$ are shown in Table I, calculated using published errors on the distance modulus data. In each case the best fit value of $a$ is found for each value of $\Omega_{m}$.

Table I lists the $\chi^{2} / N$ parameters determined for three values of $\Omega_{m}$, as well as the best fit values of $\Omega_{m}$ determined using the Mathematica software package. The latter are indicated by the word 'best' in the table. In the latter case the best fits are only statistically determined and hence also the standard error. In all instances the best fit value was determined for the parameter $a$.

From the combined data set of Riess et al and Astier et al the best statistical fit resulted in a value of $\Omega_{m}=0.0401 \pm 0.0199$, which is consistent with the result obtained by averaging the values of $\Omega_{m}$ obtained from the individual data sets.

The differences in the relative magnitudes of the $\chi^{2} / \mathrm{N}$ values for each data set is primarily the result of the size of the published errors used in the calculation (35) in the Astier et al data set. The published errors for Astier et al data are small in relation to their deviation from the fitted curve, as evidenced 
by their large $\chi^{2} / \mathrm{N}$ values compared to Riess et al data in Table I. Hence it appears that Astier et al have underestimated the real errors in their data.

Looking at the $\chi^{2} / \mathrm{N}$ values the minimum regions in each set overlap where $\Omega_{m}=0.042$. This is then the region of the most probable value. This is consistent with a value of $\Omega_{m}=0.0401 \pm 0.0199$ as determined from the combined data set shown in fig. 2. Therefore no exotic dark matter need be assumed as this value is within the limits of the locally measured baryonic matter budget $0.007<\Omega_{m}<0.041$ [13] where a Hubble constant of $70 \mathrm{~km} . \mathrm{s}^{-1} \mathrm{Mpc}^{-1}$ was assumed.

Previously one of us [14], which used some of the same data but with a different density model, the $\chi^{2} / \mathrm{N}$ parameters appear to be much smaller and therefore represent better quality fits than in the former. However this is not actually the case, as a software algorithm was used in [14] that didn't properly calculate $\chi^{2}$. The problem with the analysis was that the errors for all data were set to unity, that is, $\sigma_{i}=1$. In Oliveira and Hartnett [18] we calculated the correct $\chi^{2} / N$ parameters using (35) and published errors.

So for a comparison here, the $\chi^{2} / N$ parameters, where $\sigma_{i}$ are forced to unity, are also shown in Table I. The resulting $\chi^{2} / N\left(\sigma_{i}=1\right)$ are extremely good even compared to the 185 data of Riess et al fitted to in Fig. 1 of Hartnett [14] where $\chi^{2} / N\left(\sigma_{i}=1\right)=0.2036$ was calculated.

The improvement has resulted from the additional factor $\left(1-t^{2} / \tau^{2}\right)^{-1 / 2}$ in the luminosity distance and a little from the refinement of the density model $\Omega(z)$. If we exclude the new density model and use $\Omega=\Omega_{m}(1+z)^{3}$ where $\Omega_{m}=0.04$ instead, we get $\chi^{2} / N\left(\sigma_{i}=1\right)=0.075986$ for the best fit to the combined data set requiring $a=0.2152$. This indicates the improvement over Hartnett [14] is more the result of the additional factor in the luminosity distance than the better density model.

Looking at the curve fits of fig. 2 where the distance modulus vs redshift curves with both $\Omega_{m}=0.0401$ and $\Omega_{m}=0.263$ are shown, it is quite clear that using the Carmeli theory a universe with $\Omega_{m}=0.263$ is ruled out and hence also the need for any dark matter. This is even more obvious from the residuals shown in fig. 3 . There the fit with $\Omega_{m}=0.0401$ is drawn along the $\Delta(m-M)=0$ axis and the fit with $\Omega_{m}=0.263$ is shown as a broken line. The highest redshift data clearly rules out such high matter density in the universe.

The best fit result of this paper, $\Omega_{m}=0.0401 \pm 0.0199$, with a density function that is valid for all $z$ over the range of observations, is also consistent with the result obtained by Hartnett [14] $\Omega_{m}=0.021 \pm 0.042$ but here the $1 \sigma$ errors are significantly reduced.

With the best fit $\Omega_{m}=0.0401$, the predicted expansion transition redshift from (31) is

$$
z_{t}=1.095_{-0.155}^{+0.264} \text {. }
$$

This is about a factor of 2 greater than the fitted value reported by Riess et $a l$. [20] of $z_{t}=0.46 \pm 0.13$, which was from a best fit to the differenced distance modulus data, a second order effect. They used a luminosity distance relation assuming a flat Euclidean space (i.e., $\Omega_{\text {total }}=1$ ) and fit the difference data with 
Figure 4: Hubble constant $H_{0}$ as a function of redshift, $z$. The filled circles are determined from Tully-Fisher measurements taken from [11], Table 5 of [24] and Table 7 of [12], except the point at $\mathrm{z}=0.333$ is from Sunyaev-Zel'dovich effect taken from Fig. 4 of [24]. The open squares are determined from the SN Ia measurements and taken from Table 6 of [12] and Table 5 of [20]. The errors are those quoted in the sources from which the data was taken

the deceleration parameter $q(z)=\left(d H^{-1}(z) / d t\right)-1$.

In the present theory, the transition redshift $z_{t}$ is inherently where the density parameter $\Omega\left(z_{t}\right)=1$. Thus, the transition is determined simultaneously with the initial fit of $\mathcal{D}_{L}$ to the data.

Moreover $\Omega_{m}$ has been determined as a 'Hubble constant free' parameter because it comes from $\mathcal{D}_{L}\left(z ; \Omega_{m}\right)$, which is evaluated from fits using (34). The latter is independent of the Hubble constant or more precisely in this theory $\tau$ the Hubble-Carmeli time constant. Therefore $\Omega_{m}$ should be compared with $\Omega_{b}$ and not with $\Omega_{b} h^{2}$, where $h$ is the Hubble constant as a fraction of $100 \mathrm{~km} . \mathrm{s}^{-1} \mathrm{Mpc}^{-1}$ and not to be confused with $h=1 / \tau$ used in CGR.

Nevertheless the value of $\Omega_{b} h^{2}=0.024$ from [22] and $h=0.7217$ (assuming a value of $\tau^{-1}=72.17 \mathrm{~km} . \mathrm{s}^{-1} M p c^{-1}$ ) implies $\Omega_{b}=0.043$, which is in good agreement with the results of this work. Yet caution must be advised as the problem of the analysis of the WMAP data has not yet been attempted within the framework of CGR.

\section{$9 \quad$ Values of some key parameters}

\subsection{Hubble constant}

Using the small redshift limit of (7) and the Hubble law at small redshift ( $v=$ $H_{0} r$ ) it has been shown [7] that the Hubble parameter $H_{0}$ varies with redshift. If it applies at the low redshift limit it follows from the theory that at high redshift we can write

$$
H_{0}=h \frac{\beta \sqrt{1-\Omega}}{\sinh (\beta \sqrt{1-\Omega})} .
$$

Therefore $H_{0}$ in this model is redshift dependent, not constant and $H_{0} \leq h$. Only $h=\tau^{-1}$ is truly independent of redshift and constant. The condition where $H_{0}=h$ only occurs at $z=0$ and where $\Omega \rightarrow 0$.

By plotting $H_{0}$ values determined as a function of redshift, using (37), it is possible to get an independent determination of $h$, albeit the noise in the data is very large. This is shown in fig. 4 with values calculated by two methods with the exception of one point at $z=0.333$. See figure caption for details. The data, even though very scattered, do indicate a trending down of $H_{0}$ with redshift. 
Separate curve fits from (37), with $h$ as a free parameter, have been applied to the two data sets, Tully-Fisher (TF) (the solid line) and SNe type Ia (the broken line) measurements. The former resulted in $h=72.47 \pm 1.95$ (statistical) \pm 13.24 (rms) km.s ${ }^{-1} M p c^{-1}$ and from the latter $h=72.17 \pm 0.84$ (statistical) \pm 1.64 (rms) km.s $\mathrm{s}^{-1} M p c^{-1}$. The rms errors are those derived from the published errors, the statistical errors are those due to the fit to the data alone. The SNe Ia determined value is more tightly constrained but falls within the TF determined value.

\subsection{Mass of the universe}

It is easily shown from (27) and (28) that

$$
\Omega_{m}=R_{s} / R_{0},
$$

where $R_{s}=2 G M_{0} / c^{2}$ is the Schwarzschild radius if the present universe rest mass $M_{0}$ is imagined to be concentrated at a point, and $R_{0}=c \tau$ is the present radius of the universe. From this we get the present universe rest mass

$$
M_{0}=\Omega_{m} \frac{c^{3} \tau}{2 G},
$$

which, with $\Omega_{m}=0.0401 \pm 0.0199$ gives

$$
M_{0}=(1.74 \pm 0.86) \times 10^{21} M_{\odot} .
$$

Likewise, the average matter density (27)

$$
\rho_{m}=\Omega_{m} \rho_{c}=(3.92 \pm 1.94) \times 10^{-31} \mathrm{gm} \mathrm{cm}^{-3} .
$$

\subsection{Time of transition from deceleration to acceleration}

From Carmeli's cosmological special relativity [10] we get a relation for the cosmic time in terms of the redshift. In particular, in terms of $z_{t}$ we have for the cosmic time $t_{t}$ of the expansion transition from the present

$$
t_{t}=\tau \frac{\left(1+z_{t}\right)^{2}-1}{\left(1+z_{t}\right)^{2}+1} .
$$

For the above value of $z_{t}$ and for the age of the universe $\tau=13.58 \mathrm{Gyr}$ we have

$$
t_{t}=8.54_{-0.662}^{+0.903} \mathrm{Gyr} .
$$

Since the big bang $\left(t^{*}=0\right)$, the transition cosmic time is $t_{t}^{*}=\tau-t_{t}$,

$$
t_{t}^{*}=5.04_{-0.903}^{+0.662} \mathrm{Gyr} .
$$

In Fig. 5 is a plot of the density for $\Omega_{m}=0.04$. More than $8.54 \mathrm{Gyr}$ ago the density was higher than the critical value $(\Omega>1$.) Since the transition the density has become less than critical $(\Omega<1)$. The fit to the SNe Ia data was accomplished without the need for any dark energy, usually associated with the cosmological constant. In CGR there is no cosmological constant although a value for it may be obtained by a comparison study $[6,14]$. 
Figure 5: Density model shown as function of redshift for both approximated (broken line) and exact (solid line) with the same value of $\Omega_{m}=0.04$. The transition redshift $z_{t}=1.095$ where $\Omega=1$ is indicated by the dashed lines

\section{Conclusion}

The surface brightness is the same as in standard cosmology, though angular size is smaller by a factor of $\left(1-t^{2} / \tau^{2}\right)^{1 / 2}$.

The analysis in this paper has shown that the most probable value of the local density of the Universe is $\Omega_{m}=0.0401 \pm 0.0199$ the best fit from a combined data set of two totaling 302 data. The fits used a density function with limited range and validity and did not take into account the published errors on the individual magnitude data. The fits to the data are consistent over the entire range of the available redshift data, from $0.1<z<2.0$, a result of the more accurate relation for $\Omega$, as well as the proper accounting of the increase in the source luminosity due to the factor $\left(1-\beta^{2}\right)^{-1 / 2}$.

Since $\Omega_{m}$ is within the baryonic matter density budget, there is no need for any dark matter to account for the SNe Ia redshift magnitude data. Further-

more, since the predicted transition redshift $z_{t}=1.095_{-0.155}^{+0.264}$ is well within the redshift range of the data, the expansion rate evolution from deceleration to acceleration, which occurred about $8.54 \mathrm{Gyr}$ ago, is explained without the need for any dark energy.

The density $\Omega_{m}<1$ and the determination of the transition redshift $z_{t}$ within the data support the conclusion that the expansion is now accelerating and that the universe is, and will remain open.

\section{A Approximation of $\Omega$}

The form for $\Omega$ in (26) is transcendental, which is not convenient for fitting. A second order approximation can be made by taking $\sinh (x) \approx x+x^{3} / 3$ !. With this approximation (26) becomes

$$
\Omega \approx \Omega_{2}=\frac{\Omega_{m} / \sqrt{1-\beta^{2}}}{\left\{1-\left[\beta \sqrt{1-\Omega_{2}}+\beta^{3}\left(\sqrt{1-\Omega_{2}}\right)^{3} / 3 !\right] / \sqrt{1-\Omega_{2}}\right\}^{3}},
$$

which simplifies to

$$
\Omega_{2}\left[1-\beta-\frac{\beta^{3}}{3 !}+\frac{\beta^{3}}{3 !} \Omega_{2}\right]^{3}-\left(\Omega_{m} / \sqrt{1-\beta^{2}}\right)=0 .
$$

This is a quartic equation in $\Omega_{2}$ and can be solved for $\Omega_{2}$ as a function of $\beta$ by standard methods. $\Omega_{2}$ is shown in fig. 5 as the broken line where a matter density $\Omega_{m}=0.04$ was assumed. It is compared with $\Omega$ given by the exact form (26). 


\section{References}

[1] P. Astier et al "The Supernova Legacy Survey: Measurement of $\Omega_{M}, \Omega_{\Lambda}$ and $w$ from the first year data set," A\&A 447: 31-48 (2006)

[2] M. Carmeli, "Cosmological Special Relativity," 2nd edn., (World Scientific, Singapore, 2002)

[3] ibid. Sec. 2.15.4, p. 23.

[4] ibid. pp. 117-124

[5] ibid. pp. 125-127

[6] ibid. pp. 170-172

[7] ibid. p. 159

[8] M. Carmeli, "Cosmological Relativity: Determining the Universe by the cosmological redshift as infinite and curved", Int. J. Theor. Phys. 40: 1871$1874(2001)$

[9] M. Carmeli, "Accelerating Universe: Theory versus Experiment," arXiv: astro-ph/0205396 (2002)

[10] M. Carmeli, J.G. Hartnett, F.J. Oliveira, "The cosmic time in terms of the redshift," arXiv:gr-qc/0506079, Found. Phys. Lett. 19(3):277-283 (2006)

[11] W.L. Freedman, B.F. Madore, J.R. Mould, R. Hill, L. Ferrarese, R.C. Kennicutt Jr, A. Saha, P.B. Stetson, J.A. Graham, H. Ford, J.G. Hoessel, J. Huchra, S.M. Hughes and G.D. Illingworth, "Distance to the Virgo cluster galaxy M100 from Hubble Space Telescope observations of Cepheids", Nature 371: 757-762 (1994)

[12] W.L. Freedman, B.F. Madore, B.K. Gibson, L. Ferrarese, D.D. Kelson, S. Sakai, J.R. Mould, R.C. Kennicutt Jr, H.C. Ford, J.A. Graham, J.P. Huchra, S.M.G. Hughes, G.D. Illingworth, L.M. Macri, and P.B. Stetson, "Final results from the Hubble Space Telescope Key Project to measure the Hubble constant", Ap. J. 553:47-72, (2001)

[13] M. Fukugita, C.J. Hogan, and P.J.E. Peebles, "The cosmic baryon budget," Ap. J. 503: 518-530 (1998)

[14] J.G. Hartnett, "The distance modulus determined from Carmeli's cosmology fits the accelerating universe data of the high-redshift type Ia supernovae without dark matter," Found. Phys. 36 (6): 839-861 (2006)

[15] J.G. Hartnett, F.J. Oliveira, "Luminosity distance, angular size and surface brightness in Cosmological General Relativity," Found. Phys. 37 (3): 446454 (2007) arXiv:astro-ph/0603500 
[16] L.M. Krauss "The end of the age problem, and the case for a cosmological constant revisited," Ap. J. 501: 461-466 (1998)

[17] J.V. Narlikar, "An Introduction to Cosmology," 3rd Ed., (Cambridge University Press, Cambridge, 2002)

[18] F.J. Oliveira, J.G. Hartnett, "Carmeli's cosmology fits data for an accelerating and decelerating universe without dark matter or dark energy," Found. Phys. Lett. 19 (6): 519-535 (2006) arXiv:astro-ph/0603500

[19] S. Perlmutter et al "Measurements of the cosmological parameters $\Omega$ and $\Lambda$ from the first seven supernovae at $z>0.35, " A p . J .483: 565-581$ (1997)

[20] A.G. Riess et al "Type Ia supernovae discoveries at $z>1$ from the Hubble Space Telescope: Evidence for past deceleration and constraints on dark energy evolution," Ap. J. 607: 665-687 (2004)

[21] A.G. Riess, A. V. Filippenko, P. Challis, A. Clocchiatti, A. Diercks, "Observational evidence from supernovae for an accelerating universe and a cosmological constant", Astron. J. 116: 1009-1038 (1998)

$[22]$ D. N. Spergel et al "Wilkinson Microwave Anisotropy Probe (WMAP) three year results: Implications for cosmology," arXiv:astro-ph/0603449

[23] R.C. Tolman "On the estimation of distances in a curved universe with non-static line element," Proc. Nat. Acad. Sci. 16: 515-520 (1930)

[24] Y. Tutui et al. PASJ 53: 701, (2001) arXiv:astro-ph/0108462

[25] E.L. Wright, "Homogeneity and Isotropy; Many Distances; Scale Factor," http://www.astro.ucla.edu/ wright/cosmo_02.htm 
Table I: Curve fit parameters

\begin{tabular}{c|c|cccccc}
\hline \hline Data set & $\mathrm{N}$ & $\mathrm{a}$ & $\sigma($ stat $)$ & $\Omega_{m}$ & $\sigma($ stat $)$ & $\chi^{2} / \mathrm{N}$ & $\chi^{2} / \mathrm{N}\left(\sigma_{i}=1\right)$ \\
\hline \hline Riess et al & 185 & 0.257 & & 0.021 & & 1.34188 & \\
& & 0.268 & & 0.042 & & 1.32523 & \\
best & & 0.278 & 0.025 & 0.0631 & 0.0303 & 1.32152 & \\
\hline Astier et al & 117 & 0.158 & & 0.021 & & 11.2656 & \\
best & & 0.161 & 0.043 & 0.0279 & 0.0430 & 11.3199 & \\
& & 0.168 & & 0.042 & & 11.4533 & \\
& & 0.177 & & 0.063 & & 11.6919 & \\
\hline Riess + Astier & \multirow{3}{*}{302} & 0.219 & & 0.021 & & 6.70338 & 0.075039 \\
best & & 0.228 & 0.018 & 0.0401 & 0.0199 & 6.99446 & 0.074726 \\
& & 0.229 & & 0.042 & & 7.02192 & 0.074728 \\
& & 0.239 & & 0.063 & & 7.32371 & 0.075010 \\
& & 0.304 & & 0.263 & & 10.0568 & 0.086165 \\
\hline
\end{tabular}

